\title{
GENERATION OF PCV2 IN PK15 CELLS TRANSFECTED WITH RECOMBINANT BACULOVIRUS CONTAINING A 1.1 COPY OF THE PCV2 GENOME
}

\author{
Jie CAI, Xiaohong XIE, Yi Hu, Yang ZHAN, Wanting YU, Aibing WANG \\ and Naidong $\mathrm{WANG}^{*}$ \\ Research Center of Functional Proteomics (RCFP), College of Veterinary Medicine, \\ Hunan Agricultural University, Changsha, P. R. China
}

(Received 19 October 2016; accepted 4 April 2017)

\begin{abstract}
Porcine circovirus associated diseases (PCVAD) caused by PCV2 are responsible for severe economic losses in the swine industry. The mechanism of PCV2 replication has not been fully elucidated yet. PCV2 may be successfully rescued by means of either an infectious DNA clone containing the full length of the viral genomic DNA, or from PCV2-infected clinical tissues in PK15 cell culture. However, viruses harvested by both methods have low titres. In this study, PCV2 was prepared with a higher titre from PK15 cells infected by recombinant baculoviruses containing 1PCV2 (one stem-loop structure) or 1.1PCV2 (two stem-loop structure) genomic DNA copy. In addition, infectious DNA clones containing two stem-loop structures in either plasmid or baculovirus backbones are capable of generating a higher virus titre than the DNA clones with only one copy of stem-loop structure.
\end{abstract}

Key words: PCV2, infectious clone, stem loop structure, baculovirus, viral vector

Porcine circovirus type 2 (PCV2) is an important viral pathogen of swine (Madec et al., 2008), the causative agent of porcine circovirus associated diseases (PCVAD), which have caused severe economic losses in the global swine industry (Opriessnig et al., 2007). PCV2 is a non-enveloped single-stranded DNA virus belonging to the genus Circovirus in the family Circoviridae (Cheung, 2003b). Its ambisense circular genomic DNA (1767-1768 bases) encodes viral proteins both by the anti-sense strand for capsid protein (Cap) and by the sense strand for replication-related enzymes (Rep and Rep') (Cheung, 2012). This virus replicates its genome via a rolling-circle replication mechanism in a manner similar to the Geminiviridae family (Cheung, 2006).

Baculovirus derived from the Autographa californica nuclear polyhedrosis virus (AcMNPV) (Vail et al., 1971) has been developed as a baculovirus ex-

*Corresponding author; E-mail: naidongwang@hunau.edu.cn;

Phone/Fax: 0086 (0731) 8463-5276 
pression vector system (BEVS) and widely employed for large-scale production (in insect cells) of numerous proteins originating from either prokaryotic or eukaryotic sources (van Oers et al., 2015). Modified baculovirus (called Bacman) can express exogenous genes in mammalian cells when controlled by promoters active in those cells (Roy et al., 2013; Sung et al., 2014). The BEVS has also been used for the preparation of other viral stocks (Kotin, 2011). Adenoassociated virus (AAV) vectors produced by recombinant baculovirus and gene therapy treatment based on this vector have been registered (Haddley, 2013).

In the present study, we describe the development of a baculovirus-driven gene delivery system in the PK15 cell line for the generation of PCV2. High titres of this virus were successfully rescued from PK15 cell culture through a recombinant baculovirus system. Rescued virus was passaged serially in PK15 cells with a titre of $10^{6.5} \mathrm{TCID}_{50} / \mathrm{ml}$. This system for the preparation of PCV2 from cell culture is expected to provide a useful and robust tool for PCV2 research.

\section{Materials and methods}

Construction of 1.1 copy of PCV2 genome by overlapping polymerase chain reaction $(P C R)$

One copy of PCV2 genomic DNA was amplified from a PCV2 strain (GenBank accession number: KJ437192) using PCR with primers 1 and 2 (Table 1). Both SL-Rep (containing one stem-loop and rep gene sequence) and Cap-SL (containing cap gene sequence and one stem-loop) fragments were amplified using PCR with primers 3 and 6 , respectively (Table 1). These fragments were subsequently assembled into a 1.1 copy genomic DNA fragment (named 1.1PCV2) construct by overlapping PCR using Pfu DNA Polymerase (Transgen, China). The resultant plasmids (pSP72-1.1PCV2 and pSP72-PCV2, pFastBacI-1.1PCV2 and $\mathrm{pFastBacI-PCV2)}$ were constructed by inserting either a 1.1 or 1 copy of PCV2 genomic DNA fragment into double-digested pSP72 or pFastBacI vector (KpnI and HindIII), respectively. Ligation products were transformed into DH5 $\alpha$ competent cells and inserts confirmed by DNA sequencing.

\section{Generation of recombinant baculoviruses and monoclonal antibody}

Sf9 cells were cultured in Sf-900 II SFM insect media (Thermo Fisher Scientific, USA) at $27{ }^{\circ} \mathrm{C}$ and the Bac-to-Bac ${ }^{\circledR}$ Baculovirus Expression System was used to prepare recombinant baculovirus according to the manufacturer's instructions (Thermo Fisher Scientific, USA). PCV2 VLPs were prepared from $E$. coli in our laboratory (Zhang et al., 2016), and monoclonal antibody was produced as described previously (Walker et al., 2000). 
Table 1

Primers for plasmid construction and gene detection

\begin{tabular}{|c|c|c|c|}
\hline Product & Primer & Sequence & $\begin{array}{l}\text { Product } \\
\text { length }\end{array}$ \\
\hline \multirow[t]{2}{*}{$\begin{array}{l}\text { PCV2 } \\
\text { genome }\end{array}$} & 1 & 5'-CGGGGTACCACTGAGTCTTTTTTATCACTTCG-3'(991-1012) & $1768 \mathrm{bp}$ \\
\hline & 2 & 5'-CCCAAGCTTAAGACTCAGTAATTTATTTCATATGG(1000-975) & \\
\hline \multirow[t]{2}{*}{ SL-Rep } & 3 & $\begin{array}{l}\text { 5'-CGGGGTACCTCCTTGGATACGTCATAGCTGAAAACG-3' } \\
\text { KpnI (736-762) }\end{array}$ & $1077 \mathrm{bp}$ \\
\hline & 4 & $\begin{array}{l}\text { 5'-CCATTACGAAGTGATAAAAAAGACTCAGTAATTTATTTC } \\
\text { ATATGG-3' (1784-1759) }\end{array}$ & \\
\hline \multirow[t]{2}{*}{ Cap- SL } & 5 & $\begin{array}{l}\text { 5'-GAAATAAATTACTGAGTCTTTTTTATCACTTCGTAATGG-3' } \\
(7-35)\end{array}$ & $862 \mathrm{bp}$ \\
\hline & 6 & $\begin{array}{l}\text { 5'-CCCAAGCTTTCTTCTTGCTGGGCATGTTGCTGC-3' Hind III } \\
(850-827)\end{array}$ & \\
\hline \multirow[t]{2}{*}{ Rep } & 7 & Forward:5'-ATGACGTATCCAAGGAGGCG-3' & $945 \mathrm{bp}$ \\
\hline & 8 & Reverse: 5'-TTAGGGTTTAAGTGGG-3' & \\
\hline \multirow[t]{2}{*}{ Cap } & 9 & Forward: 5'-ATGCCCAGCAAGAAGAATGG-3' & $702 \mathrm{bp}$ \\
\hline & 10 & Reverse: 5'-TCAGTAATTTATTTC-3' & \\
\hline
\end{tabular}

Note: italics indicate restriction enzyme site, GGTACC means KpnI, AAGCTT means HindIII

\section{Generation of PCV2}

PK15 cells free of PCV1 contamination were maintained in Dulbecco's modified Eagle's medium (DMEM, Invitrogen, USA) and supplemented with $10 \%(\mathrm{v} / \mathrm{v})$ heat-inactivated fetal bovine serum (FBS, Invitrogen, USA), $100 \mu \mathrm{g} / \mathrm{ml}$ of streptomycin, and $100 \mathrm{IU} / \mathrm{ml}$ of penicillin (Invitrogen, USA). For chemical transfection, PK15 cells were seeded $\left(5 \times 10^{5}\right.$ cells/well) in 6-well plates in DMEM cultural medium and grown to approximately $60-80 \%$ confluence, and then the cells were washed with DMEM twice. Finally, cells were transfected with pSP72-1.1PCV2 or pSP72-PCV2 using Lipofectamine 2000 (Invitrogen, USA) respectively, according to the manufacturer's protocol, or infected with recombinant baculovirus (Bac-PCV2 or Bac-1.1PCV2) diluted in PBS at a multiplicity of infection (MOI) of 100 for $1 \mathrm{~h}$ (Lucifora et al., 2008). After transfection or infection, cells were washed with PBS twice, supplemented with $2 \mathrm{ml}$ of fresh medium and cultured at $37^{\circ} \mathrm{C}$ with $5 \% \mathrm{CO}_{2}$ for up to 3 days. Mock-infected cells and wild-type baculovirus infected cells were used as controls. Reverse transcription polymerase chain reaction (RT-PCR), Western blot (WB) and immunofluorescence assay (IFA) were used to detect the genome expression of PCV2 carried by baculovirus. At selected time points, supernatants were collected and frozen at 
$-80{ }^{\circ} \mathrm{C}$ or transfected cells were digested with trypsin and passaged into a new six-well plate for serial passage as described previously (Tischer et al., 1987).

\section{Detection of PCV2 cap and rep gene using RT-PCR}

Total RNA was extracted from PK-15 cells harvested $72 \mathrm{~h}$ post-transfection or infection using Trizol (Invitrogen, USA), followed by RNase-free DNase I (TaKaRa, Japan) treatment. Synthesis of cDNA s was done with RevertAid ${ }^{\mathrm{TM}}$ First Strand cDNA Synthesis Kit (MBI Fermentas, USA) with oligo (dT) 18 primer according to the manufacturer's protocol. Then PCR was performed in a $50-\mu 1$ reaction mixture with the Pfu polymerase (Transgen, France) using primers 7-10 (Table 1). The PCR products were separated on 1\% agarose gel, purified by QIAquick Gel Extraction kits (Qiagen, USA) according to the manufacturer's protocol, cloned into the pMD-18T vector by the TA cloning strategy, and sequenced.

\section{Detection of PCV2 Cap protein using Western blot}

PK-15 cells harvested $72 \mathrm{~h}$ post-transfection or post-infection were trypsinised and resuspended in $2 \times$ SDS-PAGE sample buffer in the presence of $10 \%$ beta-mercaptoethanol. Proteins were separated with 12\% SDS-PAGE and transferred onto PolyScreen PVDF membrane in a transfer chamber (Bio-Rad, Hercules, USA) according to the manufacturer's instructions. The membrane was then blocked with PBS containing 5\% bovine serum album (BSA) for $1 \mathrm{~h}$, washed three times with PBST and inoculated with an appropriate dilution of monoclonal antibody (made in our laboratory) specific to Cap for $1 \mathrm{~h}$ at room temperature. The membrane was washed three times with PBST, and then incubated with HRP-conjugated anti-mouse IgG secondary antibody (Invitrogen, USA) for $1 \mathrm{~h}$ at room temperature. Finally, the membrane was soaked in a chromogen/substrate solution (TMB signal solution, Invitrogen, USA) for colour development. Images were captured on a chemiluminescent imaging system (BioRad, USA). PCV2- (GenBank accession number: KJ437192) infected cells and wild-type baculovirus infected cells were used as controls.

\section{Immunofluorescence assay (IFA) and infectivity analysis of rescued virus}

Transfection efficiency and infectivity of rescued virus from PK15 cells transfected with pSP72-1.1PCV2 and pSP72-PCV2 or infected with Bac1.1PCV2 and Bac-PCV2 were examined by IFA. Transfection efficiency was determined $12 \mathrm{~h}$ after transfection or infection and viral infectivity was determined by inoculating PK15 cells with another passage of rescued virus. Briefly, PK15 cells were washed with PBS and fixed with $4 \%$ paraformaldehyde for $15 \mathrm{~min}$ at room temperature, followed by permeabilisation with PBS containing $0.1 \%$ Triton X-100 for $30 \mathrm{~s}$ and blocking with 1\% BSA in PBS for $2 \mathrm{~h}$. Primary antibod- 
ies against the Cap protein were used for incubation with the cells for $1 \mathrm{~h}$. After washing, secondary antibodies Alexa Fluor-488 donkey anti-mouse IgG or IgM (Invitrogen, USA) were used to facilitate visualisation. Stained monolayer cells were viewed on an Olympus IX70 inverted fluorescence microscope (Olympus, Japan). To evaluate the effects of baculovirus vectors on cell viability, wild-type baculovirus was used to infect PK15 cells as a control.

\section{Morphological observations of PCV2}

The supernatants of virus stocks after ten passages were collected and incubated with monoclonal antibodies against the Cap protein overnight at $4{ }^{\circ} \mathrm{C}$, and then centrifuged at 13,000 rpm for $30 \mathrm{~min}$. Concentrated virus was allowed to adsorb onto carbon-coated copper grids for $5 \mathrm{~min}$, then the girds were dried using filter paper and negatively stained with $3 \%$ phosphotungstic acid (PTA) for $2 \mathrm{~min}$. The samples were examined at a magnification of $\times 30,000$ using a Hitachi H500 transmission electron microscope.

\section{Viral titration of rescued PCV2}

Virus titres in cell cultures were determined by a microtitration infectivity assay recorded as $\mathrm{TCID}_{50} / \mathrm{ml}$ by using the method of Reed and Muench (Reed and Muench, 1938).

\section{Results}

Preparation of recombinant baculovirus containing 1.1 copy of PCV2 genome

The 1.1 copies of PCV2 genomic DNA were constructed from two fragments (SL-rep, 1077 bp and cap SL, 862 bp; Fig. 1A), and the resultant recombinant baculovirus contained two copies of stem loops (Fig. 1B). After amplification, the titre (plaque assay) of the baculovirus reached $1 \times 10^{8} \mathrm{pfu} / \mathrm{ml}$.

\section{Generation of PCV2 in PK15 cells infected with recombinant baculovirus}

After PK15 cells were infected with recombinant baculovirus (Bac-PCV2 and Bac-1.1PCV2), the supernatant harvested from the cell culture was reinoculated into fresh PK15 cells. Furthermore, virus stocks were propagated for 10 passages and TCID $_{50}$ was measured in each passage (Fig. 2B). We concluded that PCV2 was successfully rescued from PK15 cells infected with recombinant baculovirus containing either 1 copy of PCV2 genome or 1.1 copies of PCV2 genomes with two stem loops. As control, PCV2 was also rescued from a cell culture with plasmid transfection. It was noteworthy that the PCV2 rescued from the recombinant baculovirus (Bac-1.1PCV2) had a higher titre than others from the first passage. Regarding the morphology of PCV2 rescued from PK15 cells 
infected with recombinant baculovirus (Bac-1.1PCV2), virus stock at passage 10 had spherical particles $\sim 17 \mathrm{~nm}$ in diameter (Fig. 2A).

A

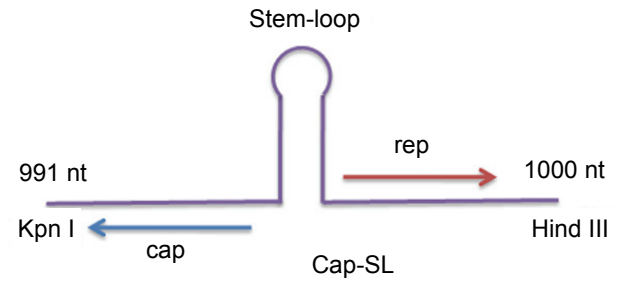

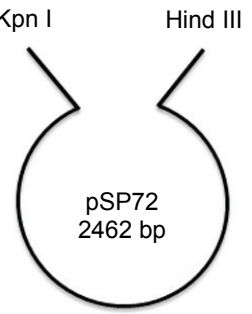
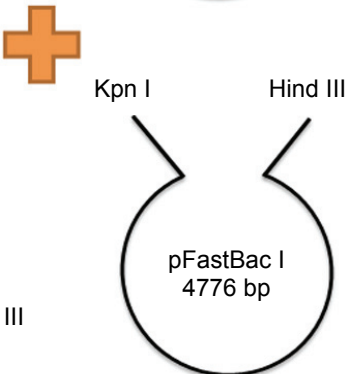

Fig. 1. Schematic diagram of plasmid construction. (A) 1 copy PCV2 genome construct. Linear full length of the PCV2 genome with one potential stem-loop structure was amplified by PCR and inserted into a pSP72 and a pFastBacI vector, respectively. (B) 1.1 copy PCV2 genome construct. 1.1 copy of PCV2 genome with two potential stem-loop structures were amplified by overlap PCR and inserted into a pSP72 and a pFastBacI vector, respectively

A

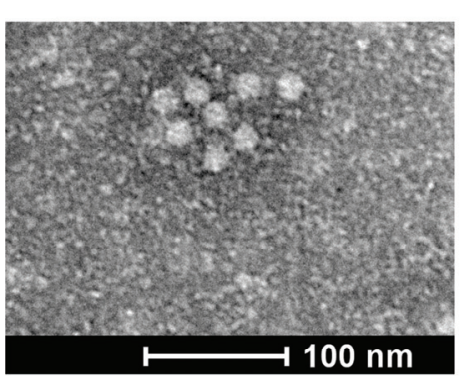

B

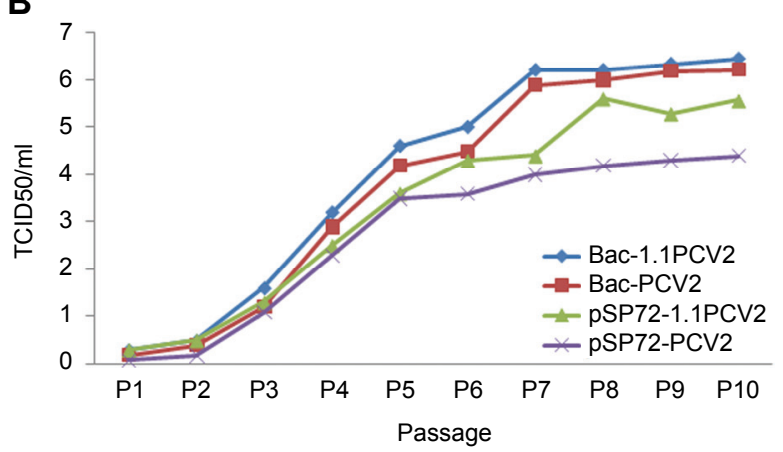

Fig. 2. Electron micrograph and viral titre of rescued virus. (A) Negatively stained PCV2 particles at passage 10 in PK15 cells infected with 100 multiplicities of infection (MOI) recombinant baculovirus; the diameter of rescued virus particles was about $17 \mathrm{~nm}$. (B) Viral titre of PCV2 in the supernatant at different passages. The titres strikingly increased at the third passage and reached $10^{6.5} \mathrm{TCID}_{50} / \mathrm{ml}$ 


\section{Characterisation of viral gene expression in PK15 cells transfected with recombinant baculovirus}

To further verify that PCV2 was rescued and propagated in PK15 cells, the expression of two major viral genes was detected by RT-PCR and subsequent DNA sequencing. As expected, specific bands of cap (702 bp) and rep (945 bp) genes were detected when total RNA was used as template. Viral cap transcripts were 10 times more than rep transcripts when using the same quantities of template RNAs (Fig. 3A, lanes 3 and 5). As control, RT-PCR was also used to detect viral gene transcripts in PK15 cells transfected with pSP72-1.1PCV2 and pSP72PCV2 (Fig. 3A, lanes 4 and 6). Wild-type baculovirus and PCV2-infected cells were used as negative control and positive control, respectively (Fig. 3A, lanes 1 and 2).

Next, expression of the capsid protein (Cap) in PK15 cells was determined $72 \mathrm{~h}$ after the cells had been infected with recombinant baculovirus (Bac-1.1PCV2 or Bac-PCV2). Based on Western blot, PCV2 Cap was detected in PK15 cells infected with recombinant baculovirus (Fig. 3B, lanes 3 and 5) or recombinant pSP72 vector (Fig. 3B, lanes 4 and 6). Wild-type baculovirus and PCV2-infected cells were used as negative control and positive control, respectively (Fig. 3B, lanes 1 and 2). Transfection efficiency was confirmed by IFA $12 \mathrm{~h}$ after infection; infected PK15 cells had nuclear staining or plasma staining with anti-PCV2 monoclonal antibodies (Fig. 4B, C, E, F). Infection with Bac-1.1PCV2 and BacPCV2 resulted in $40-60 \%$ positive cells, whereas pSP72-1.1PCV2 and pSP72PCV2 had $20-40 \%$ positive cells, with no staining apparent in mock-infected or wild-type baculovirus infected PK15 cells (Fig. 4A, D).

A

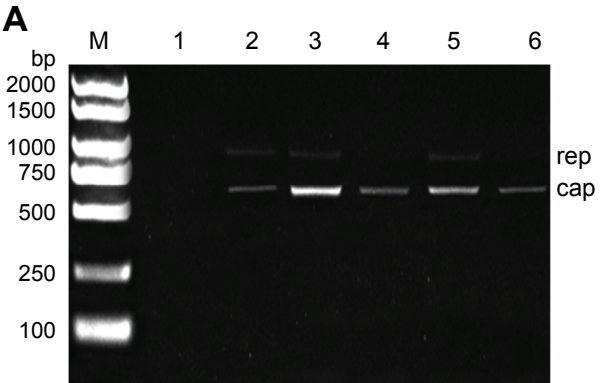

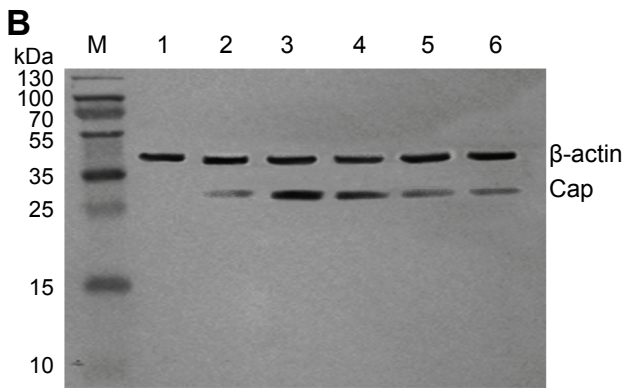

B

Fig. 3. Reverse transcription PCR and Western blot. (A) Two major viral gene transcripts were detected by reverse transcript PCR with the specific primers when the total RNA of PK15 cells infected with 100 MOI Bac-1.1PCV2 or Bac-PCV2 was used as template. (B) PK15 cells infected

100 MOI Bac-1.1PCV2 or Bac-PCV2 were harvested and total cellular protein was used in Western blot analysis with a specific antibody to structural protein Cap. Specific anti-Cap antibody recognised the $27.8 \mathrm{kDa}$ structure protein Cap of PCV2 

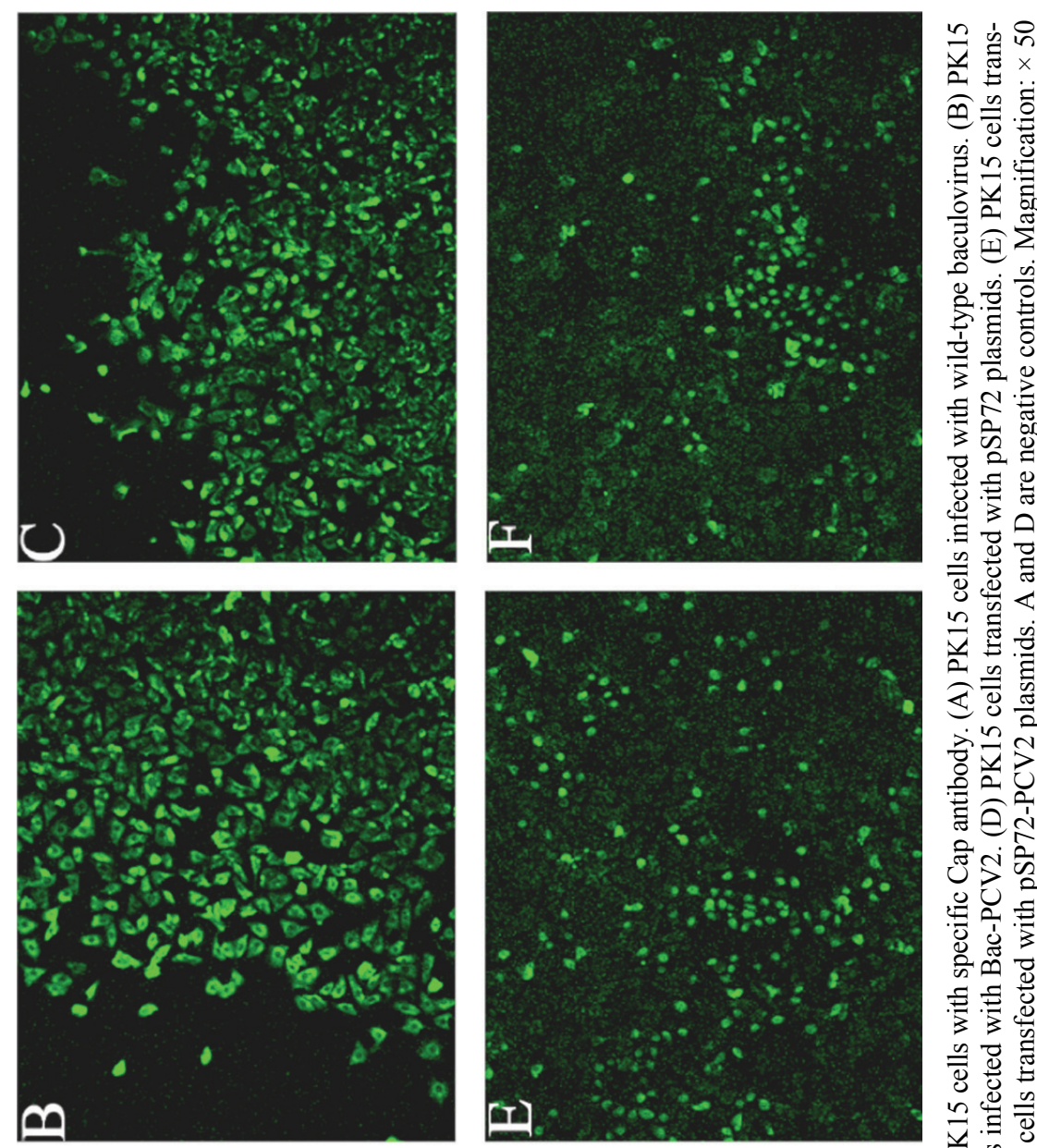

है

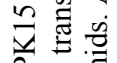

窟票

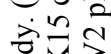

80

焉全

कैं

记

نु

की

言芦递

웛 플

魚

至
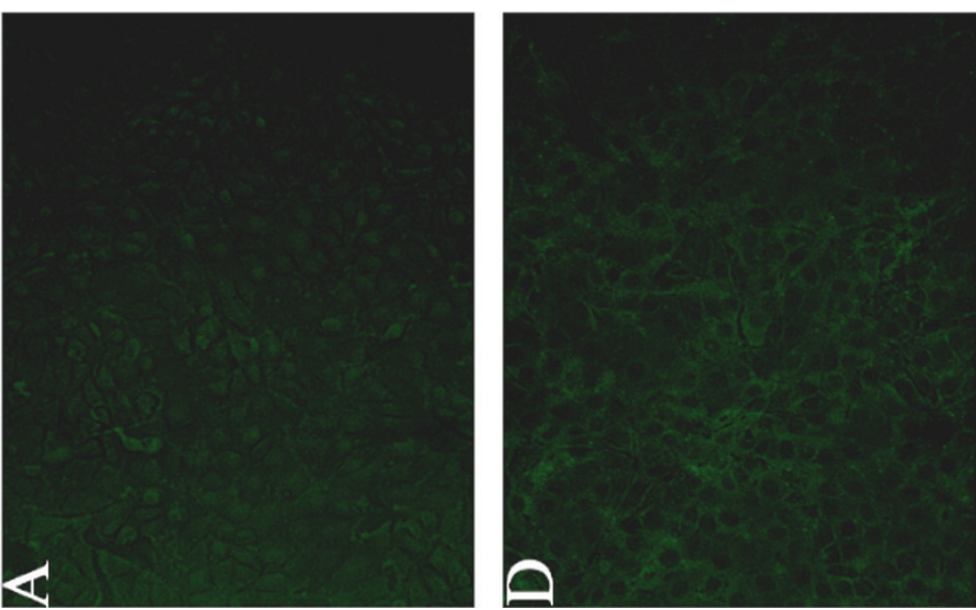

पष

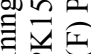

की

वृ

远

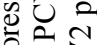

렁령

贾

罗돌

उत

兽运

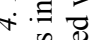

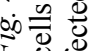




\section{Discussion}

PCV2-infected PK15 cell cultures contain only low titres $\left(\sim 10^{5}-10^{6}\right.$ $\mathrm{TCID}_{50} / \mathrm{ml}$ ) of virus (Guo et al., 2011), and consequently the development of inactivated PCV2 vaccines has been limited. In addition, PCV2 with a high titre would be useful to study PCV2 replication and for in vitro diagnosis of PCV2 infection. The preparation of a PCV2 infectious clone, a reliable method to rescue virus in the laboratory, could modify the viral genome at a molecular level, with the potential to increase PCV2 titres. To date, two laboratory-based platforms have been used to rescue PCV2. One approach is based on chemical transient transfection (Huang et al., 2012; Li et al., 2015), and the other approach used suspension cultures of invertebrate cells, such as the production of rAAV (recombinant Adeno-associated virus) vectors in insect cells (Kotin, 2011). In the present study, two PCV2 strains (Bac-PCV2 and Bac-1.1PCV2) with higher titres were rescued from recombinant baculovirus. The stability of the rescued PCV2 in PK15 cells over 10 serial passages was verified by IFA and DNA sequencing conformation. This is apparently the first report on obtaining PCV2 strains via baculovirus infection, although infectious clones for other viruses have already been rescued by baculovirus (Lucifora et al., 2008).

If two identical or similar sequences are present within one DNA molecule, this molecule may undergo homologous recombination (Valerie and Povirk, 2003; West, 2003). Furthermore, infectious virus can be generated from bacterial constructs or mammalian cells containing head-to-tail tandem repeat PCV genomes with two origins (Oris) for DNA replication (Cheung, 2006; Fenaux et al., 2002; Fenaux et al., 2003; Roca et al., 2004) or self-ligation of genomic DNA (Guo et al., 2011) upon transfection into mammalian tissue culture cells. In this study, we evaluated the role of stem-loop structure in the virus rescue. PCV2 virus strains were rescued from 1.1 copy PCV2 genome containing two stem-loop structures (pSP72-1.1PCV2 infectious clone), which yields a higher expression of viral protein and a higher titre of virus than the 1 copy PCV2 genome containing one stem-loop structure (pSP72-1PCV2 infectious clone). The rep gene mRNA transcripts essential for viral DNA replication (Cheung, 2006) of two infectious clones were detected in transfected cells by RT-PCR (Fig. 3A). The fact that the splice-joint rep' gene transcript was not detected in this study (data not shown) implies a smaller quantity of mRNA transcript, consistent with the findings of a previous study regarding transcription of PCV2 in PK15 cells (Cheung, $2003 a$ ). When this plasmid was applied in vitro, $40 \%$ of cells transfected with pSP72-1.1PCV2 had a higher expression of viral capsid antigen protein than $20 \%$ of the cells transfected with pSP72-PCV2 (based on IFA).

Rolling-circle replication (RCR), one of the most effective and simplest replication strategies, is used by several plasmids and virus (Ruiz-Masó et al., 2015). Based on the analysis of common features from the initiator Rep protein 
and stem-loop structure of PCV2, it has been proposed that PCVs, e.g. Geminiviridae and Nanoviridae, replicate using a RCR strategy (Gutierrez, 1999; Stenger et al., 1991). In the RCR mechanism, initiation and termination of DNA replication depend on a stem-loop structure (Allan et al., 2012; Cheung, 2012). A bacterial plasmid containing 1.75 copies of double-stranded porcine circovirus DNA (0.8 copy of PCV1, 0.95 copy of PCV2) in tandem with two origins of DNA replication (Ori) yielded a unit-length chimerical PCV1/PCV2 genome, which was transformed into Escherichia coli for virus rescue (Cheung, 2006). This was consistent with reports that accumulation of viral replicative-form DNAs of geminiviruses in bacteria depended on the presence of two Oris in the plasmid constructs (Selth et al., 2002). In this study, virus rescued from pSP721.1PCV2 resulted in higher PCV2 replication than pSP72-PCV2. Therefore, we inferred that the 1.1 copy PCV2 was more efficient to generate ssDNA molecule than the 1 copy PCV2. The PCV2 produced by pSP72-1.1PCV2 was likely generated via the RCR copy-release mechanism, in a manner similar to that described previously (Cheung, 2012). We inferred that PCV2 DNA synthesis was initiated at one Ori and terminated at another Ori of the same molecule. During initiation, the PCV Rep protein cleaves the PCV Oc8 sequence in one stem loop to generate a 3'-OH end for leading-stranding DNA synthesis, and polymerisation is done by mammalian replication machinery. During termination, Rep nicks another Oc8 sequence in another stem loop and then reconstitutes the Ori by joining the ends of the displaced PCV2 genome and releasing the circular ssPCV2 molecule (Cheung, 2004). Compared to RCR replication mediated two stem-loop structures (pSP72-1.1PCV2), less PCV2 DNA replication occurred in pSP72-PCV2 in spite of the same vector, perhaps because one stem-loop in pSP72-PCV2 might be less well adapted to generate a unit-length PCV2 genome. The infectious clone generated by pSP72-PCV2 was likely due to the virus recircularised by the homologous recombinant, which then jumped out of the plasmid backbone to initiate a natural infection.

The formation of recombinant baculovirus without viral replication or cytopathic effects has been used as an excellent vector to deliver and express exogenous genes in mammalian cells (Airenne et al., 2009; Sung et al., 2014) by simply adding viral inocula to these cells (Ho et al., 2005). In addition, the baculovirus technology offers an alternative to scalable virus production, due to the rapid and easy production of baculovirus, the efficient transduction of cells and the safety of baculovirus. Furthermore, baculovirus can deliver Hepatitis $\mathrm{C}$ virus (HCV) genome DNA into HepG2 cells to produce infectious HCV (Yao et al., 2008). Subsequently, functional lentivirus vectors were generated in adhered or suspended 293T cells using recombinant baculovirus (Lesch et al., 2008; Lesch et al., 2011). In this study, Bac-PCV2 and Bac-1.1PCV2 produced higher titres than pSP72-PCV2 and pSP72-1.1PCV2 in PK15 cells when infected at 100 MOI. In our study, baculovirus provided high transduction efficiency $(60 \%$ of 
the cells expressed the PCV2 viral antigen), compared to the chemical transient transfection approach (with only $40 \%$ of the PK15 cells expressing the PCV2 viral antigen). The amount of plasmids and chemical reagent determines the maximum number of transfected cells and the amount of virus produced (Guo et al., 2011), whereas the polycationic compound formed by the plasmids is toxic to the cells, which might have contributed to the low percentage of PK15 cells.

In summary, we developed an efficient and reliable baculovirus-mediated system for introducing the PCV2 genome into PK15 cells and acquiring infectious virus, which provided a superior alternative to existing production methods. In addition, we have demonstrated that the stem-loop structure was very important in virus rescue. To our knowledge, this is the first demonstration of PCV2 rescue based on recombinant baculovirus infection. Applications of this cell culture system are expected to be useful in the basic study of PCV2.

\section{Acknowledgements}

This project was supported by the General Programme of National Natural Science Foundation of China (Grants No. 31270819 and 31270819), the Hunan Provincial Natural Science Foundation of China (Grants No. 13JJ1022 and S2013J5050) and the Research Foundation of Hunan Provincial Education Department, China (Grant No. 15A086).

\section{References}

Airenne, K. J., Laitinen, O. H., Mahonen, A. J. and Yla-Herttuala, S. (2009): Transduction of vertebrate cells with recombinant baculovirus. Cold Spring Harb. Protoc. 2009, pdb prot5182.

Allan, G., Krakowka, S., Ellis, J. and Charreyre, C. (2012): Discovery and evolving history of two genetically related but phenotypically different viruses, porcine circoviruses 1 and 2 . Virus Res. 164, 4-9.

Cheung, A. K. (2003a): The essential and nonessential transcription units for viral protein synthesis and DNA replication of porcine circovirus type 2. Virology 313, 452-459.

Cheung, A. K. (2003b): Transcriptional analysis of porcine circovirus type 2. Virology 305, 168-180.

Cheung, A. K. (2004): Identification of an octanucleotide motif sequence essential for viral protein, DNA, and progeny virus biosynthesis at the origin of DNA replication of porcine circovirus type 2. Virology 324, 28-36.

Cheung, A. K. (2006): Rolling-circle replication of an animal circovirus genome in a thetareplicating bacterial plasmid in Escherichia coli. J. Virol. 80, 8686-8694.

Cheung, A. K. (2012): Porcine circovirus: transcription and DNA replication. Virus Res. 164, 46-53.

Fenaux, M., Halbur, P., Haqshenas, G., Royer, R., Thomas, P., Nawagitgul, P., Gill, M., Toth, T. and Meng, X. (2002): Cloned genomic DNA of type 2 porcine circovirus is infectious when injected directly into the liver and lymph nodes of pigs: characterization of clinical disease, virus distribution, and pathologic lesions. J. Virol. 76, 541-551.

Fenaux, M., Opriessnig, T., Halbur, P. and Meng, X. (2003): Immunogenicity and pathogenicity of chimeric infectious DNA clones of pathogenic porcine circovirus type 2 (PCV2) and nonpathogenic PCV1 in weanling pigs. J. Virol. 77, 11232-11243. 
Guo, L. J., Lu, Y. H., Huang, L. P., Wei, Y. W., Wu, H. L. and Liu, C. M. (2011): First construction of infectious clone for newly emerging mutation porcine circovirus type 2 (PCV2) followed by comparison with PCV2a and PCV2b genotypes in biological characteristics in vitro. Virol. J. 8, 291.

Gutierrez, C. (1999): Geminivirus DNA replication. Cell. Mol. Life Sci. 56, 313-329.

Haddley, K. (2013): Alipogene tiparvovec for the treatment of lipoprotein lipase deficiency. Drugs of Today (Barc.) 49, 161-170.

Ho, Y. C., Chung, Y. C., Hwang, S. M., Wang, K. C. and Hu, Y. C. (2005): Transgene expression and differentiation of baculovirus-transduced human mesenchymal stem cells. J. Gene Med. 7, 860-868.

Huang, Y. W., Patterson, A. R., Opriessnig, T., Dryman, B. A., Gallei, A., Harrall, K. K., Vaughn, E. M., Roof, M. B. and Meng, X. J. (2012): Rescue of a porcine anellovirus (torque teno sus virus 2) from cloned genomic DNA in pigs. J. Virol. 86, 6042-6054.

Kotin, R. M. (2011): Large-scale recombinant adeno-associated virus production. Hum. Mol. Genet. 20, R2-6.

Lesch, H. P., Laitinen, A., Peixoto, C., Vicente, T., Makkonen, K. E., Laitinen, L., Pikkarainen, J. T., Samaranayake, H., Alves, P. M., Carrondo, M. J., Yla-Herttuala, S. and Airenne, K. J. (2011): Production and purification of lentiviral vectors generated in 293T suspension cells with baculoviral vectors. Gene Ther. 18, 531-538.

Lesch, H. P., Turpeinen, S., Niskanen, E. A., Mahonen, A. J., Airenne, K. J. and Yla-Herttuala, S. (2008): Generation of lentivirus vectors using recombinant baculoviruses. Gene Ther. 15, $1280-1286$.

Li, P., Zhang, Z., Jia, R., Mao, S., Wang, M., Jia, R., Liu, M., Zhu, D., Chen, S. and Sun, K. (2015): Rescue of a duck circovirus from an infectious DNA clone in ducklings. Virol. J. $12,82$.

Lucifora, J., Durantel, D., Belloni, L., Barraud, L., Villet, S., Vincent, I. E., Margeridon-Thermet, S., Hantz, O., Kay, A., Levrero, M. and Zoulim, F. (2008): Initiation of hepatitis B virus genome replication and production of infectious virus following delivery in HepG2 cells by novel recombinant baculovirus vector. J. Gen. Virol. 89, 1819-1828.

Madec, F., Rose, N., Grasland, B., Cariolet, R. and Jestin, A. (2008): Post-weaning multisystemic wasting syndrome and other PCV2-related problems in pigs: a 12-year experience. Transbound. Emerg. Dis. 55, 273-283.

Opriessnig, T., Meng, X. J. and Halbur, P. G. (2007): Porcine circovirus type 2 associated disease: update on current terminology, clinical manifestations, pathogenesis, diagnosis, and intervention strategies. J. Vet. Diagn. Invest. 19, 591-615.

Reed, L. J. and Muench, H. (1938): A simple method of estimating fifty per cent endpoints. Am. J. Epidemiol. 27, 493-497.

Roca, M., Balasch, M., Segales, J., Calsamiglia, M., Viaplana, E., Urniza, A., Hattermann, K., Mankertz, A., Plana-Duran, J. and Domingo, M. (2004): In vitro and in vivo characterization of an infectious clone of a European strain of porcine circovirus type 2. J. Gen. Virol. 85, 1259-1266.

Roy, B., Liang, S., Zhang, P., Wang, M., Zhou, F., David, W. C. C. and Miao, Y. (2013): Probability to produce animal vaccines in insect baculovirus expression system. Afr. J. Biotechnol. 10, 10323-10329.

Ruiz-Masó, J. A., Machón, C., Bordanaba-Ruiseco, L., Espinosa, M., Coll, M. and Solar, G. D. (2015): Plasmid rolling-circle replication. Microbiol. Spectr. 3 (1), PLAS-0035-2014. doi: 10.1128/microbiolspec.PLAS-0035-2014.

Selth, L. A., Randles, J. W. and Rezaian, M. A. (2002): Agrobacterium tumefaciens supports DNA replication of diverse geminivirus types. FEBS Lett. 516, 179-182.

Stenger, D. C., Revington, G. N., Stevenson, M. C. and Bisaro, D. M. (1991): Replicational release of geminivirus genomes from tandemly repeated copies: evidence for rolling-circle replication of a plant viral DNA. Proc. Natl Acad. Sci. U S A 88, 8029-8033. 
Sung, L-Y., Chen, C. L., Lin, S. Y., Li, K. C., Yeh, C. L., Chen, G. Y., Lin, C. Y. and Hu, Y. C. (2014): Efficient gene delivery into cell lines and stem cells using baculovirus. Nat. Protoc. 9, 1882-1899.

Tischer, I., Peters, D., Rasch, R. and Pociuli, S. (1987): Replication of porcine circovirus: induction by glucosamine and cell cycle dependence. Arch. Virol. 96, 39-57.

Vail, P., Sutter, G., Jay, D. and Gough, D. (1971): Reciprocal infectivity of nuclear polyhedrosis viruses of the cabbage looper and alfalfa looper. J. Invertebr. Pathol. 17, 383-388.

Valerie, K. and Povirk, L. F. (2003): Regulation and mechanisms of mammalian double-strand break repair. Oncogene 22, 5792-5812.

van Oers, M. M., Pijlman, G. P. and Vlak, J. M. (2015): Thirty years of baculovirus-insect cell protein expression: from dark horse to mainstream technology. J. Gen. Virol. 96, 6-23.

Walker, I. W., Konoby, C. A., Jewhurst, V. A., McNair, I., McNeilly, F., Meehan, B. M., Cottrell, T. S., Ellis, J. A. and Allan, G. M. (2000): Development and application of a competitive enzyme-linked immunosorbent assay for the detection of serum antibodies to porcine circovirus type 2. J. Vet. Diagn. Invest. 12, 400-405.

West, S. C. (2003): Molecular views of recombination proteins and their control. Nat. Rev. Mol. Cell Biol. 4, 435-445.

Yao, X., Han, Q., Song, J., Liang, C., Wakita, T., Yang, R. and Chen, X. (2008): Baculovirus mediated production of infectious hepatitis $\mathrm{C}$ virus in human hepatoma cells stably expressing T7 RNA polymerase. Mol. Biotechnol. 40, 186-194.

Zhang, Y., Wang, Z., Zhan, Y., Gong, Q., Yu, W., Deng, Z., Wang, A., Yang, Y. and Wang, N. (2016): Generation of $E$. coli-derived virus-like particles of porcine circovirus type 2 and their use in an indirect IgG enzyme-linked immunosorbent assay. Arch. Virol. 161, 1485-1491. 